\title{
Mueller matrix differential decomposition
}

\author{
Noé Ortega-Quijano* and José Luis Arce-Diego \\ Applied Optical Techniques Group, Electronics Technology, Systems and Automation Engineering Department, \\ University of Cantabria, Avenida de los Castros S/N, 39005 Santander, Cantabria, Spain \\ *Corresponding author: ortegan@unican.es
}

Received March 9, 2011; revised April 18, 2011; accepted April 18, 2011;

posted April 19, 2011 (Doc. ID 143932); published May 13, 2011

\begin{abstract}
We present a Mueller matrix decomposition based on the differential formulation of the Mueller calculus. The differential Mueller matrix is obtained from the macroscopic matrix through an eigenanalysis. It is subsequently resolved into the complete set of 16 differential matrices that correspond to the basic types of optical behavior for depolarizing anisotropic media. The method is successfully applied to the polarimetric analysis of several samples. The differential parameters enable one to perform an exhaustive characterization of anisotropy and depolarization. This decomposition is particularly appropriate for studying media in which several polarization effects take place simultaneously. (C) 2011 Optical Society of America
\end{abstract}

OCIS codes: $260.5430,260.2130,120.5410$.

The differential Mueller calculus is a powerful technique for studying the evolution of polarized light propagation in optical media [1-3]. There are 16 basic types of optical behavior for general depolarizing anisotropic media. In a previous work, the authors presented the complete set of differential Mueller matrices corresponding to each of them [4]. They constitute a physically meaningful basis of the 16-dimensional differential Mueller space. In this Letter, we propose a Mueller matrix decomposition based on the differential calculus. It is shown that the macroscopic and differential matrices are related by their eigenvalues and eigenvectors. Therefore, it becomes feasible to obtain the differential matrix from a certain Mueller matrix and further resolve it into the basic differential matrices. As a result, any medium can be exhaustively characterized by the differential parameters, seven of them corresponding to nondepolarizing effects, and the other nine characterizing the depolarizing behavior of the sample. The decomposition is applied to several illustrative media of increasing complexity. The proposed method is validated by comparison with the results obtained by other well-known decompositions $[\underline{5}, \underline{6}]$.

First, the differential Mueller calculus is briefly summarized. We consider a beam described by the stationary statistical ensemble of the TE plane wave field. We adopt a right-handed Cartesian coordinate system [1] and assume that the beam travels toward the observer. The reference frame is set so that propagation is along the $z$ axis, and thus the field can be separated into a pair of orthogonal polarization states aligned with $x$ and $y$. The polarimetric characteristics of the beam are described by the Stokes parameters $\left\langle\boldsymbol{S}_{k=0,3}\right\rangle$ in terms of the field observables. They form the Stokes vector $\overrightarrow{\mathbf{S}}$. According to the differential formulation of Mueller calculus, the Stokes vector satisfies

$$
d \overrightarrow{\mathbf{S}} / d z=\mathbf{m} \overrightarrow{\mathbf{S}},
$$

where $\mathbf{m}$ is the $4 \times 4$ differential Mueller matrix that describes the polarimetric behavior of an infinitesimal slab of the medium [1]. The differential matrix is related to a corresponding macroscopic Mueller matrix by

$$
\mathbf{m}=\left(d \mathbf{M}_{\mathbf{z}} / d z\right) \mathbf{M}_{\mathbf{z}}^{-1} .
$$

$\mathbf{M}_{\mathbf{z}}$ is the Mueller matrix that describes the medium from $z_{0}$ to $z$. A Taylor series expansion [2] resulting from the differentiation of Eq. (2) enables one to express $\mathbf{M}_{\mathbf{z}}$ as

$$
\mathbf{M}_{\mathbf{z}}=\sum_{n=0}^{\infty}\left\{\left[\mathbf{m}^{\mathbf{n}}\left(z-z_{0}\right)^{n}\right] / n !\right\} \mathbf{M}_{\mathbf{z}}\left(z_{0}\right) .
$$

From now on, the dependence of the Mueller matrix $\mathbf{M}_{\mathbf{z}}$ on $z$ will be assumed, so it will be simply denoted as $\mathbf{M}$. If we consider a linear homogeneous medium in which $\mathbf{M}_{\mathbf{z}_{0}=\mathbf{0}}=\mathbf{I}$, the previous expression reduces to

$$
\mathbf{M}=\exp (\mathbf{m} z) .
$$

The optical properties of a general depolarizing anisotropic optical medium arise from 16 basic types of optical behavior. They span the degrees of freedom of the Mueller matrix in its entirety. In a previous paper by the same authors [4], it was demonstrated that the 16 elemental differential matrices corresponding to each single optical behavior are

$$
\begin{aligned}
& \mathbf{m}_{\mathbf{1}}=\kappa_{i} \mathbf{K}_{\mathbf{i}}=\kappa_{i} \mathbf{I},
\end{aligned}
$$

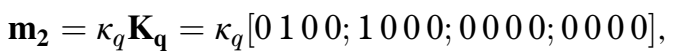

$$
\begin{aligned}
& \mathbf{m}_{\mathbf{3}}=\kappa_{u} \mathbf{K}_{\mathbf{u}}=\kappa_{u}[00010 ; 00000 ; 1000 ; 00000],
\end{aligned}
$$

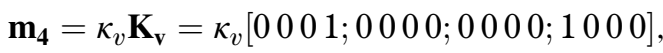

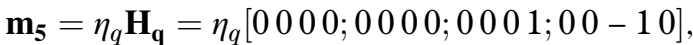

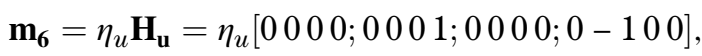

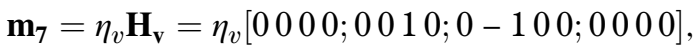

$$
\begin{aligned}
& \mathbf{m}_{\mathbf{8}}=\kappa_{i, q}^{\prime} \mathbf{D}_{\mathbf{q}}=\kappa_{i, q}^{\prime} \cdot \operatorname{diag}[0,-1,0,0],
\end{aligned}
$$




$$
\begin{aligned}
& \mathbf{m}_{\mathbf{9}}=\kappa_{i, u}^{\prime} \mathbf{D}_{\mathbf{u}}=\kappa_{i, u}^{\prime} \cdot \operatorname{diag}[0,0,-1,0], \\
& \mathbf{m}_{\mathbf{1 0}}=\kappa_{i, v}^{\prime} \mathbf{D}_{\mathbf{v}}=\kappa_{i, v}^{\prime} \cdot \operatorname{diag}[0,0,0,-1], \\
& \mathbf{m}_{11}=\kappa_{q}^{\prime} \mathbf{K}_{\mathbf{q}}^{\prime}=\kappa_{q}^{\prime}\left[\begin{array}{llllllllllllllll}
0 & 0 & 0 & 0 ;-1 & 0 & 0 & 0 ; 0 & 0 & 0 & 0 ; 0 & 0 & 0 & 0
\end{array}\right], \\
& \mathbf{m}_{\mathbf{1 2}}=\kappa_{u}^{\prime} \mathbf{K}_{\mathbf{u}}^{\prime}=\kappa_{u}^{\prime}\left[\begin{array}{lllllllllllllll}
0 & 0 & 1 & 0 ; 0 & 0 & 0 & 0 ;-1 & 0 & 0 & 0 ; 0 & 0 & 0 & 0
\end{array}\right], \\
& \mathbf{m}_{\mathbf{1 3}}=\kappa_{v}^{\prime} \mathbf{K}_{\mathbf{v}}^{\prime}=\kappa_{v}^{\prime}\left[\begin{array}{lllllllllllll}
0 & 0 & 0 & 1 ; 0 & 0 & 0 & 0 ; 0 & 0 & 0 & 0 ;-1 & 0 & 0 & 0
\end{array}\right],
\end{aligned}
$$

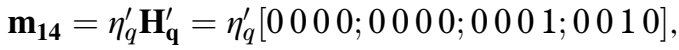

$$
\begin{aligned}
& \mathbf{m}_{15}=\eta_{u}^{\prime} \mathbf{H}_{\mathbf{u}}^{\prime}=\eta_{u}^{\prime}\left[\begin{array}{lllllllllllllllll}
0 & 0 & 0 & 0 & 0 & 0 & 0 & 1 ; & 0 & 0 & 0 & 0 & 0 & 0 & 1 & 0 & 0
\end{array}\right],
\end{aligned}
$$

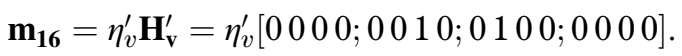

The first seven matrices correspond to nondepolarizing effects. Specifically, matrices $\mathbf{m}_{\mathbf{1 , 2 . 3 , 4}}$ describe isotropic absorption (subscript $i$ ), linear dichroism along the $x-y$ axis $(q)$ and along the bisectors of the $x-y$ axis $(u)$, and circular dichroism $(v)$, while matrices $\mathbf{m}_{5,6,7}$ account for linear $x-y$, linear $\pm 45^{\circ}$, and circular birefringence. The nondepolarizing differential parameters are directly related to the propagation constant $\tilde{\eta}=\eta+i \kappa$. We have adopted the convention $w_{q, u, v}=\left(w_{x,+45, \text { rcp }}{ }^{-}\right.$ $\left.w_{y,-45, \text { lcp }}\right) / 2$, where $w$ is either $\eta$ or $\kappa$. The nine remaining matrices correspond to depolarizing media [4]. In particular, $\mathbf{m}_{\mathbf{8 . 9 . 1 0}}$ are the differential Mueller matrices for diagonal depolarization (alternatively anomalous isotropic depolarization, i.e., polarization-dependent isotropic absorption), while $\mathbf{m}_{\mathbf{1 1 , 1 2 , 1 3}}$ and $\mathbf{m}_{\mathbf{1 4 , 1 5 , 1 6}}$ correspond to the different types of anomalous dichroism and anomalous birefringence. The differential parameters associated with the depolarizing differential matrices are given in a generic form, as long as their analytical expressions require a detailed analysis of the specific situation $[3,4]$.

The total differential Mueller matrix is the sum of the basic differential matrices [1]. Therefore, the general form of the differential Mueller matrix for depolarizing anisotropic media is

$$
\mathbf{m}=\sum_{n=1}^{16} \mathbf{m}_{\mathbf{n}}=\left[\begin{array}{cccc}
\kappa_{i} & \kappa_{q}+\kappa_{q}^{\prime} & \kappa_{u}+\kappa_{u}^{\prime} & \kappa_{v}+\kappa_{v}^{\prime} \\
\kappa_{q}-\kappa_{q}^{\prime} & \kappa_{i}-\kappa_{i, q}^{\prime} & \eta_{v}+\eta_{v}^{\prime} & \eta_{u}+\eta_{u}^{\prime} \\
\kappa_{u}-\kappa_{u}^{\prime} & -\eta_{v}+\eta_{v}^{\prime} & \kappa_{i}-\kappa_{i, u}^{\prime} & \eta_{q}+\eta_{q}^{\prime} \\
\kappa_{v}-\kappa_{v}^{\prime} & -\eta_{u}+\eta_{u}^{\prime} & -\eta_{q}+\eta_{q}^{\prime} & \kappa_{i}-\kappa_{i, v}^{\prime}
\end{array}\right] .
$$

As long as the sum of matrices is commutative, the differential matrix is order independent. The order independence of the basic differential matrices remains valid even for large sections of a medium with simultaneous optical effects [2].

Equation (1) establishes a system of four first-order linear differential equations that describes the evolution of the Stokes parameters as a function of $z$. The coefficients are given by the elements of the general differential Mueller matrix. It has been shown that the macroscopic and differential Mueller matrices are related by Eq. (2).
Their eigenvalues ( $\lambda_{\mathbf{M}}$ and $\lambda_{\mathbf{m}}$, respectively) present a similar dependence:

$$
\lambda_{\mathbf{m}}=\left(d \lambda_{\mathbf{M}} / d z\right) \lambda_{\mathbf{M}}^{-1},
$$

while, remarkably, the eigenvectors of the macroscopic and the differential matrices (grouped by columns in matrices $\mathbf{V}_{\mathbf{M}}$ and $\mathbf{V}_{\mathbf{m}}$ ) are the same [1]. We denote them simply by $\mathbf{V}$. When the initial condition $\mathbf{M}_{\mathbf{z}_{0}=\mathbf{0}}=\mathbf{I}$ is satisfied, then $\lambda_{\mathbf{M}\left(z_{0}=0\right)}=1$, and Eq. (22) can be integrated to yield

$$
\lambda_{\mathbf{m}}=\ln \left(\lambda_{\mathbf{M}}\right) / z
$$

Therefore, assuming that the macroscopic Mueller matrix is diagonalizable, it can be decomposed by a conventional eigenanalysis into

$$
\mathbf{M}=\mathbf{V M}_{\lambda} \mathbf{V}^{\mathbf{- 1}},
$$

in which the nonzero elements of the diagonal matrix $\mathbf{M}_{\lambda}$ are the eigenvalues of $\mathbf{M}$. The differential Mueller matrix can thus be obtained as

$$
\mathbf{m}=\mathbf{V} \mathbf{m}_{\lambda} \mathbf{V}^{-\mathbf{1}},
$$

where the diagonal elements of $\mathbf{m}_{\lambda}$ are the eigenvalues given in Eq. (23). Therefore, given a certain Mueller matrix $\mathbf{M}$, the corresponding differential Mueller matrix can be obtained from Eqs. (23)-(25). Once $\mathbf{m}$ is calculated, it can be easily decomposed into the physically meaningful basis of the 16-dimensional differential Mueller space formed by $\mathbf{K}_{\mathbf{i}}, \mathbf{K}_{\mathbf{q}, \mathbf{u}, \mathbf{v}}, \mathbf{H}_{\mathbf{q}, \mathbf{u}, \mathbf{v}}, \mathbf{D}_{\mathbf{q}, \mathbf{u}, \mathbf{v}}, \mathbf{K}_{\mathbf{q}, \mathbf{u}, \mathbf{v}}^{\prime}$, and $\mathbf{H}_{\mathbf{q}, \mathbf{u}, \mathbf{v}}^{\prime}$ [implicitly given in Eqs. (5)-(20)]. The decomposition of $\mathbf{m}$ according to the general form of Eq. (21) thus yields the 16 differential parameters associated with each basic optical effect, namely $\kappa_{i}, \kappa_{q, u, v}, \eta_{q, u, v}, \kappa_{i, q}^{\prime}, \kappa_{i, u}^{\prime}$, $\kappa_{i, v}^{\prime}, \kappa_{q, u, v}^{\prime}$, and $\eta_{q, u, v}^{\prime}$. It should be noted from Eq. (23) that the determination of the differential matrix from an experimentally measured Mueller matrix requires one to know the value of $z$, i.e., the optical path undergone by the measured photons, which is not an obvious issue in many applications. If $z$ is unknown, the optical pathweighted differential matrix $\overline{\mathbf{m}}=\mathbf{m} z$ will be obtained instead of $\mathbf{m}$. Therefore, it involves accumulated effects. In this situation, the decomposition of $\overline{\mathbf{m}}$ results in accumulated differential parameters, which are denoted with an upper bar.

The Mueller matrix differential decomposition described above is now particularized for several media in order to verify the proposed method. In particular, we demonstrate the decomposition for Mueller matrices of different homogeneous media measured in transmission configuration. Nonhomogeneous media and backscattering measurements will be discussed in future works. The first one is a nematic liquid crystal plate. An example measured Mueller matrix is [7]

$$
\mathbf{M}_{1}=\left[\begin{array}{cccc}
1.0000 & 0.0622 & -0.0038 & 0.0023 \\
0.0646 & 0.9888 & -0.1407 & -0.0468 \\
-0.0026 & -0.1227 & -0.5846 & 0.7988 \\
0.0015 & -0.0829 & -0.7838 & -0.6029
\end{array}\right] .
$$


The accumulated differential Mueller matrix corresponding to this sample is

$$
\overline{\mathbf{m}}_{\mathbf{1}}=\left[\begin{array}{cccc}
-0.0020 & 0.0625 & -0.0011 & -0.0006 \\
0.0644 & -0.0022 & -0.0260 & 0.1843 \\
-0.0063 & 0.0255 & 0.0054 & 2.2264 \\
0.0036 & -0.1824 & -2.1931 & -0.0158
\end{array}\right] .
$$

The decomposition of this matrix into the complete basis described above yields $\bar{\eta}_{q}=2.2097$ and $\bar{\eta}_{u}=0.1833$. The other parameters are very close to zero, which asserts that it is a birefringent nondepolarizing sample. From these values, we obtain that the linear retardance is $2.2175 \mathrm{rad}$, which is identical to the result derived by other methods [7].

We now demonstrate the decomposition on a depolarizing medium. The measured Mueller matrix of a piece of adhesive [7] in transmission configuration is

$$
\mathbf{M}_{2}=\left[\begin{array}{cccc}
1.0000 & 0.0056 & 0.0019 & 0.0064 \\
0.0081 & 0.8461 & -0.0028 & 0.0169 \\
0.0037 & -0.0002 & 0.7672 & 0.3358 \\
0.0048 & -0.0216 & -0.3485 & 0.7728
\end{array}\right] .
$$

The differential decomposition of this matrix presents four dominant components. Three of them correspond to depolarizing accumulated differential parameters $\bar{\kappa}_{i, q}^{\prime}=0.1668, \bar{\kappa}_{i, u}^{\prime}=0.1747$, and $\bar{\kappa}_{i, v}^{\prime}=0.1676$, and the last one is $\bar{\eta}_{q}=0.4181$. The rest of parameters are negligible. Therefore, the analysis indicates that it is a linear birefringent medium with nearly polarization-independent isotropic depolarization. In order to compare these results with the macroscopic depolarization power involved in polar decomposition [5], we obtain the macroscopic depolarizing matrix from the differential depolarizing parameters mentioned above, and we calculate the depolarization power of this matrix, which is found to be 0.1561 . The calculated total linear retardance is $23.99^{\circ}$. These values fully coincide with the results obtained by polar decomposition [7].

Once those relatively simple media have been studied, we analyze a turbid medium with several simultaneous effects. The measured Mueller matrix of a birefringent chiral turbid sample [ㅇ] is

$$
\mathbf{M}_{3}=\left[\begin{array}{cccc}
1.0000 & 0.0185 & 0.0029 & 0.0042 \\
0.0172 & 0.7569 & -0.0405 & 0.0462 \\
0.0034 & 0.0524 & 0.5450 & -0.5466 \\
0.0024 & -0.0070 & 0.6244 & 0.5967
\end{array}\right] .
$$

The sample is a tissue phantom that shows a scattering coefficient of $30 \mathrm{~cm}^{-1}$ and an anisotropy parameter of 0.95 at $633 \mathrm{~nm}$, with a $1 \mathrm{M}$ concentration of sucrose characterized by an optical activity parameter of $\chi=$ $1.96^{\circ} / \mathrm{cm}$ and linear birefringence induced by vertical strain. The polarimetric analysis of this sample has been performed using polar decomposition and is reported elsewhere [8]. The control values are a linear retardance
Table 1. Accumulated Differential Parameters of the Tissue Phantom Characterized by the Matrix in Eq. (29)

\begin{tabular}{ccccccccc}
\hline \multicolumn{8}{c}{ Nondepolarizing Parameters } \\
\hline \multicolumn{10}{c}{$\bar{\kappa}_{i}$} & $\bar{\kappa}_{q}$ & $\bar{\kappa}_{u}$ & $\bar{\kappa}_{v}$ & $\bar{\eta}_{q}$ & $\bar{\eta}_{u}$ & $\bar{\eta}_{v}$ & \\
0.000 & 0.021 & 0.003 & 0.003 & -0.799 & 0.036 & -0.067 & \\
\hline \multicolumn{8}{c}{ Depolarizing Parameters } \\
\hline $\bar{\kappa}_{i, q}^{\prime}$ & $\bar{\kappa}_{i, u}^{\prime}$ & $\bar{\kappa}_{i, v}^{\prime}$ & $\bar{\kappa}_{q}^{\prime}$ & $\bar{\kappa}_{u}^{\prime}$ & $\bar{\kappa}_{v}^{\prime}$ & $\bar{\eta}_{q}^{\prime}$ & $\bar{\eta}_{u}^{\prime}$ & $\bar{\eta}_{v}^{\prime}$ \\
0.276 & 0.236 & 0.167 & 0.001 & -0.001 & 0.002 & 0.052 & -0.001 & -0.007 \\
\hline
\end{tabular}

of $0.83 \mathrm{rad}$, optical rotation of $0.0373 \mathrm{rad}$, depolarization power of 0.19 , and null diattenuation, while the calculated values from the measured matrix are respectively 0.79 and $0.0357 \mathrm{rad}, 0.21$, and 0.02 . If we apply the differential decomposition to this sample, the parameters graphically shown in Table $\underline{1}$ are obtained. The analysis reveals that the medium presents linear retardance along the $y$ axis, polarization-dependent isotropic depolarization, and optical activity. It also has marginal anomalous birefringence along the $x$ axis and linear birefringence along the $x-y$ bisector. The remaining contributions are negligible. The linear retardance is found to be $0.8022 \mathrm{rad}$, while the calculated net optical rotation is $0.0338 \mathrm{rad}$. The depolarization power is 0.2016 , and a value of 0.0210 for total diattenuation is obtained. All the results are in excellent agreement with the previously reported values.

These results validate the differential Mueller matrix decomposition proposed in this Letter. This decomposition is particularly appropriate for the study of media in which several effects take place simultaneously, as long as no assumption about the order in which effects take place has to be made aprioristically. In fact, differential decomposition constitutes the first unique Mueller matrix decomposition. We believe this method can definitely help overcome the ambiguities entailed by the nonunique Mueller matrix product decompositions proposed so far $[\underline{5,6}, \underline{9}]$. The exhaustive description performed by the 16 differential parameters enables one to develop detailed studies about the optical properties of complex media, with a foreseeable potential for a wide range of applications.

\section{References}

1. R. M. A. Azzam, J. Opt. Soc. Am. 68, 1756 (1978).

2. D. S. Kliger, J. W. Lewis, and C. E. Randall, Polarized Light in Optics and Spectroscopy (Academic, 1990).

3. C. Brosseau, Opt. Lett. 20, 1221 (1995).

4. N. Ortega-Quijano and J. L. Arce-Diego, "Depolarizing differential Mueller matrices," Opt. Lett. (to be published).

5. S. Y. Lu and R. A. Chipman, J. Opt. Soc. Am. A 13, 1106 (1996).

6. J. Morio and F. Goudail, Opt. Lett. 29, 2234 (2004).

7. F. Boulvert, G. Le Brun, B. Le Jeune, J. Cariou, and L. Martin, Opt. Commun. 282, 692 (2009).

8. N. Ghosh, M. F. G. Wood, S. Li, R. D. Weisel, B. C. Wilson, R. Li, and I. A. Vitkin, J. Biophoton. 2, 145 (2009).

9. R. Ossikovski, J. Opt. Soc. Am. A 26, 1109 (2009). 